\title{
In vino veritas: Cultura, educazione e enoturismo nel contesto d'innovazione
}

\author{
Eliane Schlemmer $^{1, \mathrm{a}}$, Wagner dos Santos Chagas ${ }^{1, \mathrm{a}}$, Cleber Portal ${ }^{1, \mathrm{a}}$, et Bruno Cisilotto ${ }^{2, \mathrm{~b}}$ \\ ${ }^{1}$ Universidade do Vale do Rio dos Sinos Programa de Pós-Graduação em Educação, São Leopoldo, Brasil \\ ${ }^{2}$ Instituto Federal de Educação, Ciência e Tecnologia do Rio Grande do Sul Departamento de Enologia, Bento Gonçalves, Brasil
}

\begin{abstract}
The paper presents In Vino Veritas - IVV, a hybrid, pervasive and ubiquitous game, developed in Bento Gonçalves, located in Serra Gaúcha, Southern Brazil. The goal is to discuss the cultural, educational and wine tourism potential of the IVV, in a context of innovation, including learning in the areas Enology, Gastronomy and History of Italian immigration. The conception and development methodology were inspired by the concept of Living Lab (William J. Mitchell) and the cartographic method of intervention-research (Virgínia Kastrup) As instruments were used participant observation, photographic records, audio, text, digital video and interviews. The data produced were analyzed and interpreted from the Actor-Network Theory (Bruno Latour). The results point to the innovation initiatives such as the IVV might contribute in the field of culture, education and wine tourism, articulated through a narrative able to engage tourists in experiences of knowledge, mixing geographical and digital spaces, valuing local culture.
\end{abstract}

\begin{abstract}
Sommario. L'articolo presenta lo In Vino Veritas - IVV, un gioco ibrido, pervasivo e onnipresente, sviluppato nella comune di Bento Gonçalves, situata nella regione della Serra Gaúcha nel sud del Brasile. L'obiettivo di questo articolo è quello di discutere il potenziale culturale, educativo e enoturistico del IVV, nel contesto di innovazione, tra cui l'apprendimento nelle aree di enologia, gastronomia e storia dell'immigrazione italiana. La metodologia di progettazione e sviluppo sono stati ispirati dal concetto di Living Lab (William J. Mitchell) e il metodo cartografico della ricerca-intervenzione (Virginia Kastrup). Mentre gli strumenti, sono stati utilizzati, osservazione partecipante, documentazione fotografica, audio, testo, video digitale e interviste. I dati prodotti sono stati analizzati e interpretati dalla teoria Actor- Network (Bruno Latour). I risultati indicano l'innovazione che iniziative come il IVV può portare nel campo della cultura, dell'educazione, e dell'enoturismo, articolato attraverso una narrazione in grado di coinvolgere turisti in esperienze di conoscenza, mescolando gli spazi geografici e digitali, valorizzando la cultura locale.
\end{abstract}

\section{Introduzione}

Gli ambienti attuali in cui gli esseri umani vivono e convivono, ogni volta sono più come ibridi quanto riguarda la natura degli spazi (digitale e geografico), la presenza (fisica e digitale), la tecnologia (analogica e digitale) e cultura (analogico, digitale, gamer, maker, tra gli altri). Questo ibridismo è potenziato da multimodalità dove coesisti la presenza fisica e on- line; la pervasività e onnipresenza che consentono l'apprendimento situato, pervasivo, fornendo le informazioni al soggetto "sensibili" al proprio profilo, necessità, ambiente e altri elementi del contesto di apprendimento, ovunque si sia e in qualsiasi momento. Questo è possibile attraverso le tecnologie di posizionamento (GPS, sistemi di navigazione, di localizzazione di persone); tecnologie di identificazione (tag RFID e QR Code, marcatori); sensori, tra gli altri, che possono essere presenti in varie applicazioni, giochi e processi di gamificazione (ludicizzazione). È in questo

a e-mail: elianeschlemmer@gmail.com; tutorwagnergp@ gmail.com; cleber portal@gmail .com

b e-mail: bruno.cisilotto@bento.ifrs.edu.br contesto che gli attuali soggetti di apprendimento si sviluppano, formando la propria cultura, in congruenza con questo spazio-tempo storico- sociale.

Consapevoli di questa realtà, il gruppo di ricerca in educazione digitale - GPe-dU UNISINOS/CNPq e il Instituto Federal de Educação de Bento Gonçalves, sostenuta da CAPES, CNPq, FAPERGS e ABED, insieme dell'Azienda Vinicola Aurora, Azienda Vinicola Dal Pizzol, Azienda Vinicola Larentis, Azienda Vinicola Miolo, Azienda vinicola Geisse, Ristorante Canta Maria, Casa da Erva Mate, Itallinni Biscotteria, Casa da Ovelha e Casa do Tomate e da Gasosa, hanno costituito una collaborazione che ha reso possibile la costruzione del in Vino Veritas IVV.

L'IVV nasce dalla necessità di esplorare nuove possibilità d'istruzione, cultura e turismo, attraverso la ricerca del potenziale d'ibridazione, multimodalità, pervasività e onnipresenza, in combinazione con i giochi e la gamificazione, includendo la città come uno spazio che integra l'apprendimento, favorendo esperienze di conoscenza. L'obiettivo è quello di discutere il potenziale culturale, educativo e enoturistico dell'IVV, in un contesto 
di innovazione, che include l'apprendimento nelle aree di Enologia, Gastronomia e storia dell'immigrazione italiana.

\section{Materiali e metodi}

La ricerca, che dà luogo all'IVV è di naturalezza esplorativa e approccio qualitativo. La metodologia per la ricerca e lo sviluppo, è stato ispirato dal concetto di Living $L a b$, proposto da William J. Mitchell e, nello metodo cartografico di ricerca-intervento, proposto da Kastrup (2007, 2008), Passos et al. (2009), Passos et al. (2014), con cui abbiamo cartografato il processo costruito nello sviluppo dell'ARG e nella ricerca.

Living Labs è un concetto che nasce negli anni '90 e si riferisce a un ecosistema di innovazione aperta, collaborativa, che è concentrata nel soggetto nel contesto della ricerca pubblico-privato (imprese, governo, università, centri tecnologici), di solito legata a un contesto territoriale. L'idea principale è quella di co-creazione di processi di ricerca e innovazione, per i soggetti che vivono e convivono in dato contesto territoriale. Così, l'IVV comprende diversi attori umani nel quadro della ricerca pubblica-privata, in collaborazione di cocreazione del processo di ricerca e innovazione. Questa co-creazione ha coinvolto una squadra interdisciplinare a diversi livelli di processi di ricerca e innovazione, da esplorazione, a la sperimentazione e la valutazione di idee e concetti emergenti fino a partecipazione alla progettazione, sviluppo, monitoraggio e valutazione dell'idea, del concetto e dell'artefatto tecnologico. Il concetto coinvolge quindi i soggetti/comunità come attori nel processo di creazione della conoscenza e non solo come osservatori o utenti. Così, un laboratorio vivente è costituito da un ambiente esperienziale (sperimentale + empirico), in cui i soggetti sono immersi in uno spazio sociale creativo per concepire, progettare, monitorare e valutare il proprio futuro. Questo concetto è collegato al cambiamento e trasformazione sociale.

In questo contesto, abbiamo utilizzato il metodo di mappatura di ricerca-intervento (Passos et al., 2009) (Passos et al., 2014) destinato a seguire il processo. L'attenzione cartografica è definito come concentrato e aperta, caratterizzata da quattro movimenti che compongono l'attenzione del cartografo: il rintracciamento, il tatto, l'atterraggio e il riconoscimento attento, che ha coinvolto e hanno definito come il ARG doveva funzionare. L'obiettivo del cartografo.

è proprio la mappatura del territorio producendo conoscenza attraverso un percorso di ricerca, che coinvolge l'attenzione, e con essa, la creazione stessa del territorio di osservazione. (KASTRUP, 2007, p. 2007). Così, per essere ricerca-intervento, l'analisi avviene nel processo, nel movimento della mappatura, che consente di eseguire l'intervento mentre il processo è in corso.

Come gli strumenti sono stati utilizzati osservazione partecipante, registri di foto, audio, testi, video digitale e interviste semi-strutturate. I dati sono stati analizzati e interpretati dal quadro teorico presentato di seguito.

\section{Giochi ibridi, pervasive e onnipresente}

I giochi sono presenti nella vita quotidiana dei bambini, adolescenti, giovani, adulti, anziani, di entrambi i sessi, che contribuisce alla nascita della cultura Gamer. Secondo Schwartz (2015) uno studio sul settore dei giochi digitali in Brasile e nel mondo, guidati dal PGT-FEA- USP per il BNDES nel 2014, ha concluso che:

Gli usi di giochi digitali hanno superato l'intrattenimento, incorporando attività di formazione, la ricerca scientifica, la formazione (a livello aziendale e anche per la difesa nazionale), e anche usufruire di formazione connesse alle cure sanitarie nella scelta e nello sviluppo di vocazioni, e l'architettura e la costruzione (p. 165).

Ma a chi si attribuisce la crescita di questa dimensioni? Cosa possiamo imparare dalla cultura Gamer? I giochi impegnano il soggetto in un mondo di finzione, immaginazione e fantasia, consentendo l'interattività, immersione, narrativa, agenzia, divertimento e trasformazione, tra gli altri elementi che contribuiscono a questo impegno. Diventa significativo per i giocatori, soprattutto perché permettono a loro di vivere un'esperienza (trasformare le informazioni in esperienza). Collegato a questo contesto sono movimenti come "Games for Change", il cui obiettivo è l'uso di giochi per il cambiamento sociale. Secondo McGonigal (2011) se i giocatori sono disposti ad intraprendere le sfide che coinvolgono gli ostacoli, spesso inutili, significa che hanno la capacità di mobilitare e potrebbero essere utilizzati come strumento di trasformazione sociale.

I giochi possono essere analogici, digitali, o ibridi. L'ibrido è capito da Latour (1994), mentre comprendente più matrici, miscele di cultura e natura e quindi nessuna separazione tra cultura/natura, umana/non umana, ecc. Cioè, è le azioni e le interazioni tra attori umani (presenti in forma fisica e digitale) e non-umani (in aree di natura geografica e digitali attraverso tecnologie analogiche e digitali) in un intreccio di culture diverse (pre-digitale e digitale - gamer, maker, tra gli altri), che costituiscono un fenomeno inseparabili, reti che collegano la natura, le tecniche e le culture. La pervasività e onnipresenza presumono che l'informazione sono disperse nello spazio e nelle cose/oggetti, che collega spazi geografici e spazi digitali, e si può accedere da dispositivi mobili, collegati alle reti wireless. Così, essi possono migliorare l'insieme di apprendimento situato, pervasivo fornendo al soggetto le informazioni "sensibili" al proprio profilo, le esigenze, l'ambiente e altri elementi del contesto di apprendimento, ovunque e in qualsiasi momento. Così, le tecnologie di posizionamento (GPS, sistemi di navigazione, di localizzazione di persone); tecnologie di identificazione (tag RFID e QR Code, marcatori); sensori, tra gli altri, possono essere presenti in varie applicazioni, giochi e processi di gamificazione. L'ubiquità (onnipresenza) collabora per integrare gli discenti di apprendimento e loro contesti circostanti, permettendo formare una rete fisica e virtuali digitale tra le persone, oggetti, situazioni o eventi. Relativi alla mobilità,

Pervasività e ubiquità ci sono anche la "realtà mista" (RM) e la "realtà aumentata" (RA), che combinano una scena di spazio geografico, visto da un soggetto con una scena digitale e, nel caso di realtà aumentata, le informazioni digitali aggiunge alla scena geografica ingrandendola, vale dire "aumentare la scena", migliorando la conoscenza degli oggetti, luoghi o eventi. Sia RM o RA sono concetti diversi e loro hanno diversi tipi di 
configurazioni, ma entrambi consistono principalmente nel riconoscimento di un oggetto, denominato "marcatore", progettato in un ambiente geografico, una fotocamera che cattura questo oggetto e un software specifico in grado di ricevere le informazioni inviate dalla telecamera, interpretarle e progettare le informazioni digitali sull'oggetto di spazio geografico.

È in questo contesto teorico che è stato sostenuto lo sviluppo di IVV, capito come un gioco ibrido, pervasivo e onnipresente, che può essere caratterizzato come un ARG.

\section{IVV: II Proggetto e il gioco nel contesto della cultura del enoturismo e innovazione}

L'IVV, dal concetto di Living Lab, emerge da un processo di co-creazione interdisciplinare all'interno della ricerca pubblico-privato (aziende, associazioni e università), con la partecipazione di diversi attori umani in diversi momenti del processo di ricerca e innovazione, legati all'area territoriale della città di Bento Gonçalves. È in questo contesto che nasce la prospettiva del turismo culturale, che comprende le "attività turistiche legate alla esperienza del insieme di elementi significativi del patrimonio storico e culturale, e di eventi culturali, con la valorizzazione e promozione dei beni materiali ed immateriali della cultura" (MTur, 2006, p.13). Si propone di fornire ai visitatori esperienze positive con il patrimonio storico e culturale della regione. Queste esperienze avvengono nel contesto di interazione con gli elementi costitutivi del percorso turistico culturale di esperienze partecipative e contemplative che forniscono l'interpretazione, la comprensione e l'apprezzamento della cultura locale. Collegato al turismo culturale, nel contesto di Bento Gonçalves, ci sono anche i concetti di turismo gastronomico e del enoturismo.

Il turismo gastronomico, secondo il Ministero del Turismo (2010), corrisponde a l'itinerari di visita tra ristoranti e altri spazi che rappresentano le tradizioni culinarie della regione, dove è stabilito un legame emotivo con la cultura locale attraverso esperienze sensoriali risvegliate dai cibi caratteristici della regione. Così l'atto di degustare qualche prodotto gastronomico ha il potenziale per guidare il turista per un viaggio nel tempo e nello spazio di varie culture attraverso il loro repertorio alimentare. Già il enoturismo, secondo il Ministero del Turismo (2010), è l'attività turistica che mira a conoscere il processo di produzione del vino, spumanti e derivati, visitando le cantine e vigneti, dove i turisti possono degustare e acquistare i prodotti legati alla produzione di vino. In Brasile le prime iniziative che hanno esplorato la cultura della produzione di uva e vino come attrazione turistica è apparso nella regione sud del Brasile in cui le cantine hanno aperto i loro stabilimenti al pubblico. In queste visite $\mathrm{i}$ turisti percorrono l'itinerario di visita seguito l'intero processo di produzione del vino. Quindi, il turismo è alimentato dal movimento di riconoscimento e di apprezzamento della storia e della cultura delle comunità locali, aggiungendo valore alla intera catena di produzione del vino, così come la generazione di nuove opportunità per la creazione di reddito per gli abitanti di questa regione.

Il turismo culturale, gastronomico e lo enoturismo può essere migliorato attraverso l'IVV, istigando visite ai diversi punti di Bento Gonçalves. Queste visite rappresentano un "giro" turistico con la presentazione e la conservazione del carattere culturale degli oggetti, così come il monitoraggio delle attività quotidiane, eventi tradizionali della comunità locale, luoghi di interesse storico e culturale, ristoranti, aziende vinicole, e altri che rappresentano le tradizioni della regione.

Quindi, considerando questo concetto, il progetto IVV si è sviluppato dal problema e gli obiettivi espliciti precedentemente, con la collaborazione dei seguenti aziende: Casa da Ovelha, Casa da Erva-Mate, Casa do Tomate e da Gasosa, Cantina Canta Maria, Itallinni Biscotteria, Azienda Vinicola Aurora, Azienda Vinicola Dal Pizzol, Azienda Vinicola Larentis, Azienda Vinicola Miolo, Azienda Vinicola Geisse e a Azienda Vinicola do Instituto Federal de Bento Gonçalves. I collaboratori sono stati scelti in base alle potenzialità del turismo culturale e le caratteristiche specifiche (concetti) che hanno individualizzato e evidenziato la potenzialità di ciascun collaboratore, dove hanno servito anche come guida per la creazione delle missioni.

Dalla definizione e il contatto iniziale con i collaboratori, ha cominciato le visite per presentare il progetto ed effettuare lo invito. Nella misura in cui hanno accettato il progetto anche ha iniziato a comporre il metodo cartografico della ricerca intervento, con l'esplorazione dello spazio, dai quattro movimenti che compongono l'attenzione del cartografo

- lo tracciato, l'atterraggio, il tatto e il riconoscimento attento. Lo tracciato (esplorazione/scansione degli spazi geografici e on-line da ciascuna delle societàpartner, alla ricerca di indizi - informazioni per comprendere $\mathrm{i}$ processi), il tatto (selezione di indizi geografiche, tracce online e brani dal vivo, per comporre la narrazione e missioni), l'atterraggio (zoom sulle piste, scegliere/impostazione della narrazione e il concetto di ogni missione differenziale rispetto ai processi e dei prodotti e la visita agli spazi locali) e il riconoscimento attento (come funzionerà il ARG?).

Gli indizi trovati e trasformati in missioni, dai movimenti che compongono l'attenzione del cartografo, costituite come l'integrazione di spazi di apprendimento nelle aree di Enologia, Gastronomia, chimica e storia, intesi come elementi per lo sviluppo della cultura e della cittadinanza. In questo contesto, gli strati informativi erano legati ai ricordi personali e collettivi o dell'immigrazione italiana, suscitate dai sensi, impegnando i soggetti in un mondo di sensazioni e scoperta culturale.

Fatto questo primo ciclo del metodo cartografico è stato avviato il processo di sviluppo dell'applicativo IVV (disponibile per il sistema operativo IOS e Android), un ARG che fa uso di meccaniche e dinamiche presente nei giochi di MMORPG - Massive Multiplayer Online Role Play Game e dispone di identità visiva e gli elementi caratteristici della storia dell'immigrazione italiana, gastronomia e produzione di uve e vino. Il giocatore può interagire in quattro lingue: portoghese, inglese, spagnolo e italiano.

L'IVV ha una narrazione iniziale, organizzata in tre atti: $1^{\circ}$ Atto, Mitologia - Bacco, toccato dalla vita quotidiana dura degli esseri umani, regala alla umanità la capacità di produrre il vino; $2^{\circ}$ Atto, Immigrazione 
italiana - sfide e le difficoltà incontrate da immigrati italiani durante il processo di immigrazione per il Brasile; $3^{\circ}$ Atto, Immigrati in Bento Gonçalves - Regalo di Bacco della produzione del vino e tutti gli elementi materiali e immateriali della cultura italiana a Bento Gonçalves. Questo racconto spinge i giocatori per realizzare le missioni, a cui si accede dalla mappa, presenti nel gioco, attraverso le icone vino e spazi gastronomici presenti nel gioco. Tre personaggi in 3D - Bacco, nona Francesca e lo italiano Guiuseppe continuano la narrazione, spingendo il giocatore a svolgere i compiti, che implica il movimento in città e l'interazione con la comunità locale, la costruzione di una rete di conoscenze, distribuita in undici missioni. Quando si sceglie un collaboratore (missione), il giocatore apre una schermata in cui ha la sintesi della missione, le informazioni del locale (orari di apertura, numeri di telefono, ecc.), posizione e un filmato - un video che mostra un poco del locale della missione. Oltre a queste informazioni, il giocatore ha la possibilità dicliccare sul "come arrivare", che si indirizza al Google Maps, che riceve le informazioni di percorsi per raggiungere il locale della missione prescelta.

$\mathrm{Al}$ suo arrivo presso il posto della missione, il giocatore ha bisogno di trovare i marcatori che contiene "Indizi Online" - RM e RA e "Indizi Vive" - le persone collegate al posto, riferimento alle conoscenze necessarie per sviluppare la missione. Quindi, per iniziare il percorso di gioco, il giocatore deve selezionare l'icona della lente di ingrandimento e punto al marcatore, rendendo la lettura. In quel momento uno dei personaggi presenti nel gioco interagirà con lui, guidando per lo cammino della missione. In questo cammino il giocatore può venire attraverso diverse possibilità che vanno da interagire con un Indizi Vive, trovare un prodotto specifico, assaggiare un cibo, fare qualche attività specifiche del locale, ecc. Mentre il giocatore sta svolgendo le missioni si espanderà il suo livello di EXP o XP, in altre parole, il livello di esperienza (conoscenza). Il gioco ha quattro livelli di esperienza: Umano, Eroe, Semidio e Dio. Per ogni tre missioni completate il giocatore aumenta il loro livello di esperienza.

Quando si eseguono le missioni i giocatori possono condividere esperienze, sensazioni, intuizioni, dare suggerimenti, fare critica e interagire con gli altri giocatori in un gruppo creato su Facebook, cioè come spazio collaborativo. Queste informazioni sono analizzate da un gruppo di esperti e può comporre nuovi versioni del gioco. Così l'IVV costituisce un gioco ibrido multimodale pervasivo e onnipresente, può anche essere cooperativo, in quanto i giocatori hanno l'opportunità di diventare co-autori, socializzando la conoscenza costruita sull'interazione con le aree geografiche e gioco digitale.

\section{Resulalti e discussioni}

I dati generati nella ricerca-sviluppo-ricerca sono stati sistematizzati ed organizzate sottosistemi di informazione (con l'aiuto di Excel) come contesto (progettazione, sviluppo, monitoraggio e valutazione - che coinvolge le prove, l'osservazione dei giocatori e interviste con i giocatori e imprese-collaboratrice), dal quale è emerso le categorie di analisi presentata al software di analisi dei dati NVivo. Le categorie sono state suddivise in teorica (attante, intermedio, traduzioni, iscrizione, scatola nera, lo spazio-tempo, polemiche, immersione, agenzia, la trasformazione, l'impegno) ed empirica (curiosità, novità, l'innovazione, la giocosità, l'interazione, l'apprendimento, la strategia, valutazione).

Le categorie di attante, intermedio, di traduzione, di iscrizione, scatola nera, spazio- temporali, controversia sono legati ai diversi contesti, mettendo in evidenza la robustezza del TAR per aiutare nella comprensione dei dati, per sottolineare la partecipazione di non umano

- oggetti e quasi oggetti (dispositivi mobili, il app IVV, oggetti diversi e aree geografiche), le relazioni sociali, dal momento che anche interferire negli eventi, e nella creazione di significato - che agisce sulla sfera riflettente e simbolica. Dal momento che i diversi attanti (AH - compresso come tutte le persone coinvolte nel processo del gioco, tra cui designer, sviluppatori, ricercatori, insegnanti, lettori, turisti, uomini d'affari, indizi vive, tra gli altri, e, ANH compresso come gli oggetti , sistemi, linguaggi, luoghi, dispositivi mobili, marcatori, processi, tra gli altri) sono stati identificati come produttori del movimento associative (relazioni), generatori di azione, che "fatto fare", dunque, proprietari di agenzie, che mostrano il punto di vista della "vasta simmetria "o" ontologia piatta". Capire il punto di vista sociale, è stato dato a seguire gli attori (mappatura) nelle loro associazioni e riassociazione (stabilito come movimenti nomadi in ambienti ibridi, multimodali pervasivo e onnipresente) che formano le reti.

La categoria di immersione, agenzia, la trasformazione e l'impegno associati con la prospettiva d'ibridismo, multimodalità, pervasività e ubiquità, sono stati osservati in diversi contesti, tuttavia, in modo più efficace durante i test e, successivamente, in seguito di gruppi di turistigiocatori, dove è stato possibile percepire che il gioco ha fornito un maggiore impegno dei turisti-giocatori con il luogo e la comunità, in base alla coerenza tra i diversi spazi che costituivano il gioco (applicativo, località geografiche, indizi vive), che sono stati tracciati dalla narrazione che ha fomentato la conoscenza e la vicinanza, sia con le tecnologie digitali, analogiche o persone stesse. In questo momento, che si stabilisce una affinità comune o rapport, un continuum è presentato, dove il turistagiocatore rimane completamente assorbito, immerso nella situazione cambiando la loro percezione del tempo stato di flow. Questa prospettiva è stata confermata dai risultati trovati durante l'analisi d' interviste condotte con i giocatori, i turisti e con i collaboratori che hanno accompagnato il processo.

La categorie curiosità, novità, l'innovazione, la giocosità, l'interazione, l'apprendimento, la strategia, la valutazione, è emerso dall'empirismo, durante lo sviluppo dei diversi contesti di cui summenzionato, particolarmente evidente nei momenti di interazione con le aziende partner tra $\mathrm{i}$ registro in audio e video e nel interviste semistrutturata, e nella comunità creata su Facebook per l'IVV, attraverso registro di testo.

Queste categorie hanno dimostrato la potenzialità del gioco, di pensare a nuove possibilità di coinvolgere meglio i turisti alla comunità e i luoghi visitati, in modo che possano dare più senso alle informazione, vivendo 
l'esperienza di conoscenza che coinvolgono enologia, gastronomia, storia dell' immigrazione italiana, ridefinendo le forme di appropriazione delle zone turistiche della città.

I risultati mostrano che le azioni e le interazioni tra attori umani e non umani sono stati resi possibili dal IVV, attraverso l'ibridazione di spazi geografici e digitali, in un intreccio di culture diverse, con la loro legittimazione. In questo contesto, la multimodalità ha dato un carattere di continuità e l'estensione delle azioni e interazioni nel tempo e nello spazio, per consentire la costruzione della conoscenza che si sviluppa nella sovrapposizione di modalità presenziale con la modalità online attraverso il gioco pervasivo e onnipresente che coinvolti missioni giocate in diverse parti della città portando alla cattura di informazioni dall'ambiente/persone così come la cattura e/o produzione di geo-informazioni, utilizzando la RM e RA, nonché interazione nel gruppo creato su Facebook per la condivisione di esperienze tra $\mathrm{i}$ giocatori.

Questa costruzione ci aiuta a capire che ibridismo, multimodalità, la pervasività e ubiquità è stato favorevole a la creazione di spazi di convivenza che legittimano attori umani e non umani nel processo di apprendimento, tra cui la città e le sue socialità.

\section{Conclusioni}

L'IVV, dal momento del concepimento, ha avuto la preoccupazione di propiziare l'interazione della tecnologia con le aree geografiche e la comunità locale. Così, a partire dal obiettivo di questo articolo che è stato quello di discutere il potenziale culturale, educativo e enoturistico dell'IVV, in un contesto di innovazione, che include l'apprendimento nelle aree di Enologia, Gastronomia e storia dell'immigrazione italiana, possiamo dire che il gioco IVV ha fornito una riformulazione di itinerari turistici attraverso l'intreccio tra spazi geografici e digitali. Questo rete ha potenziato immersione turistico-giocatori nella cultura delle comunità, esperienze che valorizzano le radici storiche della colonizzazione italiana nella città di Bento Gonçalves. Questo apprezzamento delle radici storiche della cultura italiana permea dalla tavola con gli elementi del cibo, il processo di crescita dell'uva e la produzione di vino, spumante e succo d'uva; al riconoscimento dell'importanza della conservazione del patrimonio storico e culturale immateriale, presente nei racconti orali di persone che compongono queste comunità. Così il progetto IVV è un differenziato turistico alternativo di mobilità-giocatori intorno alla città che si trasforma in uno spazio esteso di apprendimento, in grado di sviluppare un nuovo rapporto tra il patrimonio materiale e immateriale storico e culturale e le persone che ricontestualizzano questo patrimonio. Con questo l'IVV potenzia lo sviluppo di esperienze coinvolgenti nella cultura locale attraverso il contatto con le attività tradizionali che fanno parte dell'atmosfera di itinerario enoturistico e turismo gastronomico.

\section{Riferimenti Bibliografici}

Bertoco, C., 2014. Sustentabilidade, planejamento urbano e instrumentos de gestão do patrimônio e da paisagem cultural em Bento Gonçalves/RS. 2014. Monografia. Universidade de Brasília, Brasília

Brasil, 2006. Segmentação do Turismo: Marcos Conceituais. Brasília: Ministério do Turismo

Brasil, 2010. Ministério do Turismo. Turismo Cultural: orientações básicas. 3.ed. Brasília: Ministério do Turismo

Kastrup, V., 2007. O funcionamento da atenção no trabalho do cartógrafo. Psicologia \& Sociedade, 19 (1), p. 15-22. Disponível em: <http://www.scielo. $\mathrm{br} / \mathrm{pdf} / \mathrm{psoc} / \mathrm{v} 19 \mathrm{n} 1 / \mathrm{a} 03 \mathrm{v} 19 \mathrm{n} 1 . \mathrm{pdf}>$

Kastrup, V., 2008. O método cartográfico e os quatro níveis da pesquisa-intervenção. In: Pesquisaintervenção na infância e adolescência. Castro, L. R.; Besset, V. (orgs). Rio de Janeiro: Nau editora, p. 465-489

Latour, B., 1994. Jamais fomos modernos. Rio de Janeiro: Editora 34

Latour, B., 2012. Reagregando o social: uma introdução à teoria do ator-rede. São Paulo: EDUSC

Lemos, A., 2013. A comunicação das coisas: teoria atorrede e cibercultura. São Paulo: AnnaBlume

Mcgonigal, J., 2012. A realidade em jogo. Rio de Janeiro: Best Seller

Passos, E. et al., 2009. Pistas do método da cartografia: pesquisa-intervenção e produção de subjetividades. Porto Alegre: Sulina

Passos, E. et al., 2014. Pistas do método da cartografia: a experiência da pesquisa e o plano comum. Porto Alegre: Sulina

Saccol A., et al., 2011. M-learming e u-learning - novas perspectivas da aprendizagem móvel e ubíqua. São Paulo: Pearson

Schlemmer, E., 2014. Gamificação em espaços de convivência híbridos e multimodais: design e cognição em discussão. Revista Faeeba, v. 23, p. 73-89

Schlemmer, E., 2015. Gamificação em contexto de hibridismo e multimodalidade na educação corporativa. Revista FGV Online, v. 5, p. 26-49

Schlemmer, E., 2016. Gamificação em espaços de convivência híbridos e multimodais: uma experiência no ensino superior. Relatório Técnico de Pesquisa. Ciências Humanas, Sociais e Sociais Aplicadas

Schlemmer, E., Backes, L., 2015. Ambientes imersivos, realidade misturada, realidade aumentada, gamificação, ECODI e ECHIM. 1. ed. São Leopoldo: Unisinos. Schlemmer, E., Backes, L., 2015. Learning in Metaverses: Co-existing in Real Virtuality. 1. ed. Hershey: IGI Global, v.1

Schwartz, G., 2015. Cidade do conhecimento e a iconomia dos jogos com moedas criativas. Revista Organicom, p. 160-179, v. 12 